\title{
Tumoriform Focal Muscular Degeneration in Two Diabetic Patients
}

\author{
By \\ L. Angervall and B. Stener \\ From the Departments of Pathology II and Surgery I, Sahlgrenska sjukhuset, University of Gothenburg \\ Received March 13, 1965
}

Summary. Focal muscular degeneration in two diabetics is described. In both instances the muscular lesion was excised on the grounds of clinical suspicion of tumour. The gross and microscopical pictures agreed with those of muscular degeneration following ischaemia. Accordingly, the vascular lesions that were demonstrated, diabetic microangiopathy and arteriosclerosis, may have been of importance in the pathogenesis.

Résumé. Une dégénérescence musculaire focale a été observée chez deux diabétiques. Dans les deux cas la lésion musculaire a été excisée, une tumeur ayant été considérée possible. L'apparence macroscopique et microscopique était celle d'une dégénérescence musculaire consé-

Focal muscular degeneration in two diabetic patients will be described. In both instances the muscular lesion was excised on the grounds of elinical suspicion of a tumour. Such focal muscular degeneration, presenting clinically as tumoriform, seems to be little known. The histological examination showed diabetic microangiopathy and arteriosclerosis, i.e. vascular changes that may have been of importance for the development of the muscular degeneration.

\section{Case Reports}

Case 1. A man born in 1907 was found to have diabetes and hypertension in 1949. Intermittently during the next decade he had albuminuria as well. The diabetes was treated dietetically, the hypertension with various agents including hydralazine. Toward the end of 1959 the back of the right thigh became painful and swollen without known trauma.

At examination four weeks after onset, palpation revealed at the site of biceps femoris a firm, circumscribed, elongated mass, with its long axis (a bit over $10 \mathrm{~cm}$ ) parallel to the muscle fibres. The mass was mobile when the thigh muscles were relaxed, but became fixed when the knee flexors contracted. A tumour was suspected. Femoral angiography, performed to elucidate the nature of the mass, failed to establish the presence or absence of a tumour, but disclosed axteriosclerosis in large and medium-sized arteries in the thigh. The blood pressure was 260/145. No injections had been given in the affected region.

At operation the mass proved to be located within the biceps femoris muscle, which was extirpated without prior excision of a biopsy specimen (loss of this muscle leads to negligible impairment of function).

Gross examination of the extirpated muscle showed at the site of the mass a well circumscribed, dark reddish-brown foeus which felt firmer than the rest of cutive à une ischémie. Nous avons donc considéré que les lésions vasculaires démontrées, microangiopathie diabétique et artériosclérose, ont probablement contribué à la pathogénèse de ces dégénérescences musculaires.

Zusammenfassung. Bei zwei Diabetikern werden umschriebene muskuläre Degenerationsherde geschildert. In beiden Fällen wurden die krankhaft veränderten Muskelgebiete wegen des klinischen Verdachtes auf Tumor exzidiert. Die makro- und mikroskopischen Befunde waren die gleichen wie bei Muskeldegeneration nach Ischämie. Demnach waren für die Pathogenese möglicherweise die nachgewiesenen Gefäßveränderungen, die diabetische Mikroangiopathie und Arteriosklerose, von Bedeutung.

the muscle (even though it included small soft regions). After transection, the musculature within the focus did not retract as did the perifocal musculature, but protruded on the cut surface.

Histological examination (Fig. 1) revealed within the circumscribed focus, regions of haemorrhagic

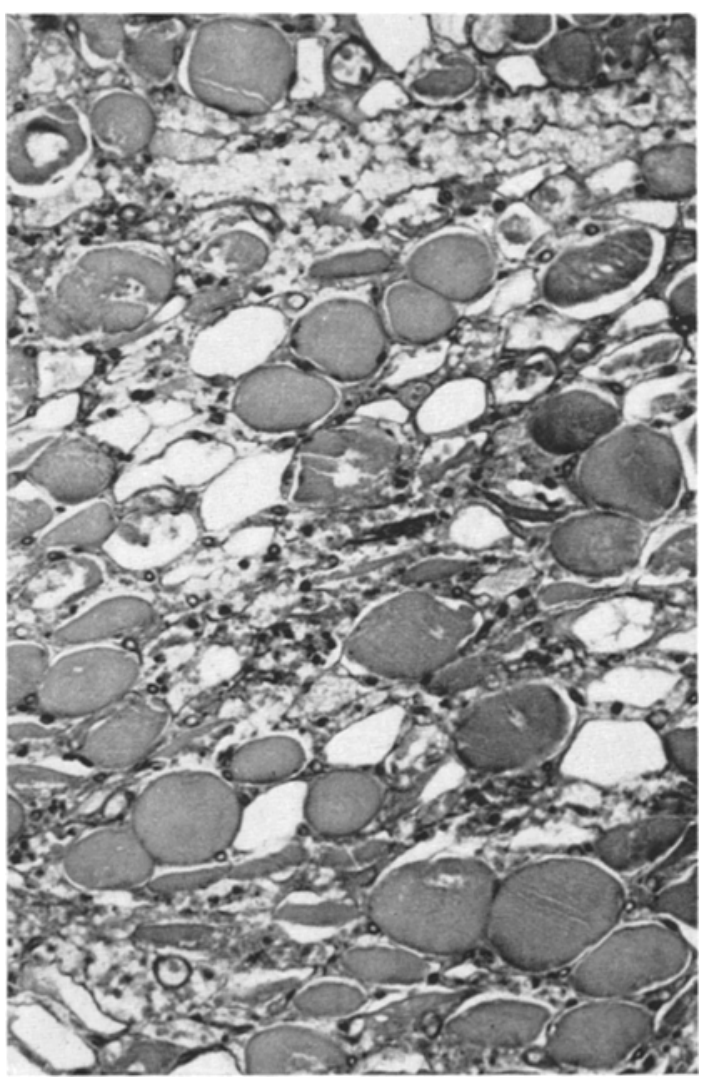

Fig. 1. Case 1. From necrotic region within focus. Note capillaries with thickened walls and appearing as dark (strongly PAS stained), round, "rigid" rings of varying calibres. (PAS staining according to McManus, $\times 192$ ) 
necrosis surrounded by muscle tissue that exhibited regressive changes and evidence of regeneration. In necrotic regions, the muscle fibres were homogeneous, unstructured (the striation lost), some shrunken, others swollen; many of them, with hyperchromatic nuclei,

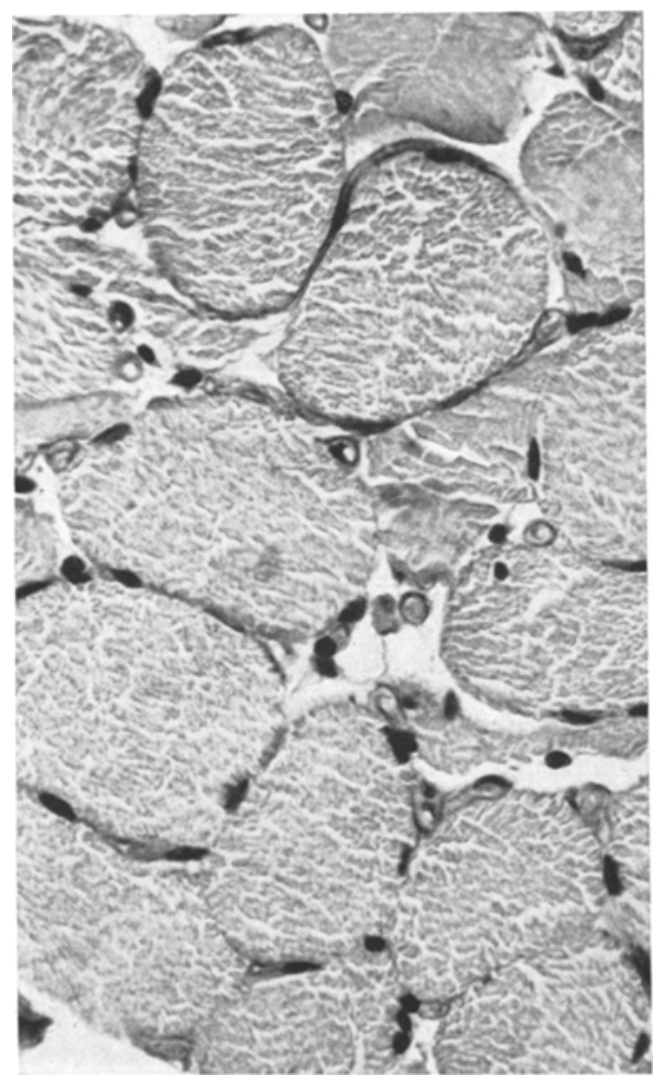

Fig. 2. Case 1. Perifocal intact muscle tissue. Capillaries with thickned, PAS stained walls appear as dark, round, "rigid" rings. (PAS staining according to McManus, $\times 480$ )

were strongly acidophilic; the endomysium was increased in width, oedematous, and exhibited merely residues of necrotic or necrobiotic reticulum, infiltrated by a few neutrophilic leukocytes. Around necrotic regions, regressively altered muscle fibres, with striation only partly preserved, presented here and there univacuolar or multivacuolar degeneration; the sarcolemma nuclei were hypertrophic, increased in number, and displayed prominent nucleoli; occasionally, vacuolized sarcoplasm was encircled by rings of sarcolemma nuclei. The degenerated muscle fibres were to a large extent separated by a highly vascular connective tissue, rich in fibroblasts.

The vessels within the circumscribed focus were pathologically changed: the walls of capillaries, arterioles and small arteries were thickened and often hyalinized, and in PAS stained sections (McManus 1948) they showed a pronounced PAS staining; some vessels, within necrotic regions, were partly necrotic. The thickening of the walls had greatly reduced and occasionally obliterated the lumen of the capillaries.
Intraneural capillaries also exhibited the above changes. Some arteries showed proliferation of the intima. Similar but less pronounced vascular changes were observed in the intact musculature outside the focus (see capillaries with thickened, PAS stained walls in Fig. 2). Here, the largest arteries showed extensive calcification of the media (MöncKEBERG's arteriosclerosis).

Further clinical course. After the operation the patient was put on insulin. He remained at work (as a custom's clerk) until the spring of 1961, when progressive gangrene of the right foot necessitated amputation of the lower leg; he was discharged as soon as the stump had healed. In September 1961 he was admitted to another hospital for cardiac decompensation and nephropathy. (Ophthalmoscopy revealed hypertensive and diabetic retinopathy.) In this hospital, he died suddenly in January 1962, after myocardial infarction.

Case 2. A man born in 1929 was found to have diabetes in 1957, for which he was treated with tolbutamide. In the middle of December 1959, the patient - although no known trauma had occurred began to have a dull pain in the left thigh, particularly at night. He noticed a tender lump laterally in the thigh and two weeks later another one medially.

At examination four weeks after onset, palpation of the middle part of the thigh revealed an approximately $2 \mathrm{~cm}$ broad, elongated mass (parallel to the muscle fibres) in the vastus lateralis, and an ovoid mass measuring 3 by $5 \mathrm{~cm}$ in the adductor muscles. Both masses were firm and well demarcated. Femoral angiography revealed at the sites of the masses a faint accumulation of contrast medium of uncertain diagnostic significance. Since the masses were entirely separate, and indistinguishable from tumours at palpation, it was suspected

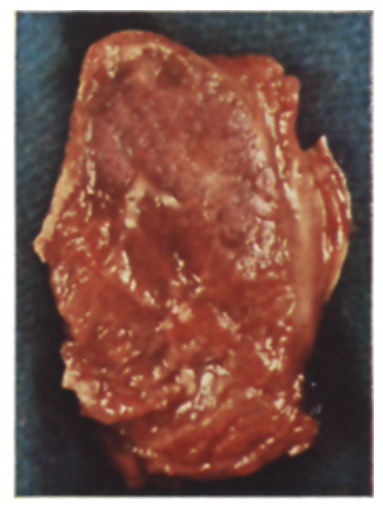

Fig. 3. Case 2. The cut surface of the operative specimen. Focus in the upper part. (Natural size)

that they were metastases from a cryptogenic malignant tumour. The blood pressure was 130/95-90. No injections had been given in the thigh.

Operation. The lateral mass, located in vastus lateralis, was excised together with surrounding normal muscle tissue (extirpation biopsy). 
Grossly the excised portion of vastus lateralis contained a fusiform, well circumscribed though unencapsulated focus, which crackled during transection. The cut surface of the focus (Fig. 3) was reddish-brown and firmer than ordinary muscle tissue.

Histological examination (Fig. 4) disclosed within the circumscribed focus, regions of haemorrhagic necrosis surrounded by muscle tissue in which the muscle fibres were atrophic, but also showed signs of regeneration. In necrotic regions, unstructured, homogeneous muscle fibres were found separated by wide, oedematous spaces filled with a necrotic or necrobiotic reticulum, invaded by neutrophilic leukocytes. In atrophic muscle fibres the sarcolemma nuclei were increased in number, hypertrophic and rich in chromatin. Highly vascular granulation tissue occurred here and there, invaded by lymphocytes and plasma cells.

Within the focus, and to a lesser extent outside it, thickening of the vascular wall was observed in capillaries and arterioles. The finest capillaries were often

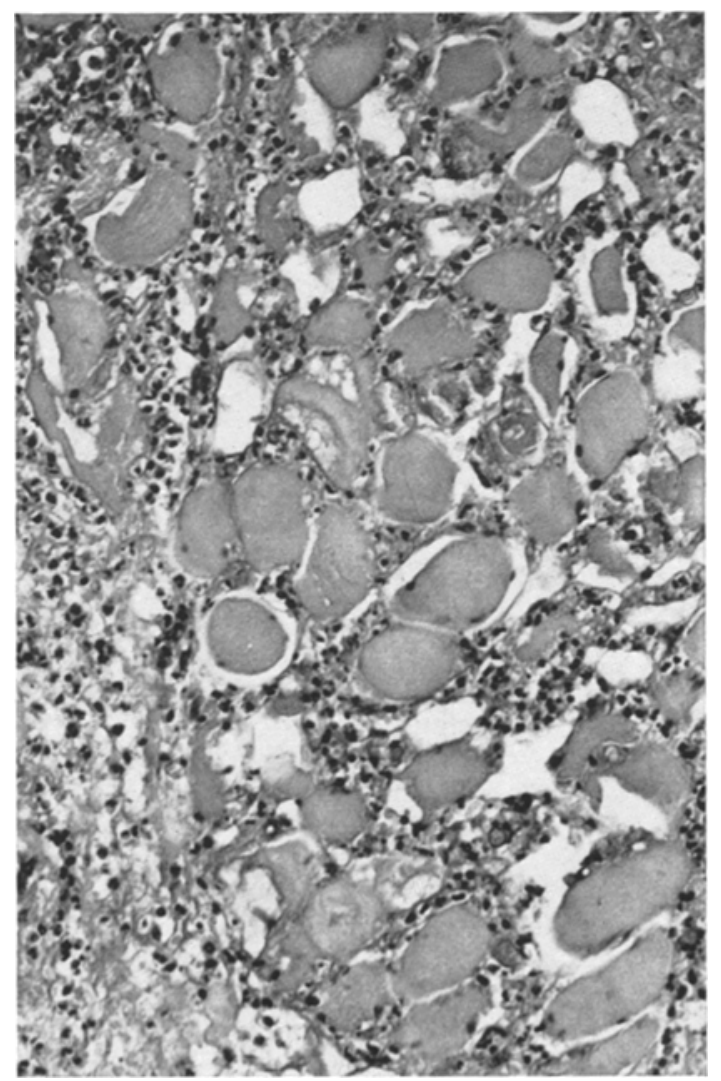

Fig. 4. Case 2. Muscle necrosis within focus. The degenerated muscle fibres are separated by wide, oedematous spaces filled with a necrotic or necrobiotic reticulum, invaded by neutrophilic leukocy tes. (PAS staining according to MoManus, $\times 192$ )

strongly PAS-positive (Fig. 5). The walls of the coarsest capillaries and arterioles exhibited concentric, PASpositive lamellas. At the boundary between intact and abnormal muscle tissue, intraneural capillaries were encountered that had thickened, PAS stained walls. Some of the largest arteries showed atheromatosis.

Further clinical course. After the operation the patient was put on insulin. Assumed to be similar to the excised one, the mass medially in the thigh was left untouched and disappeared spontaneously. There has been no recurrence during an observation time of four years.

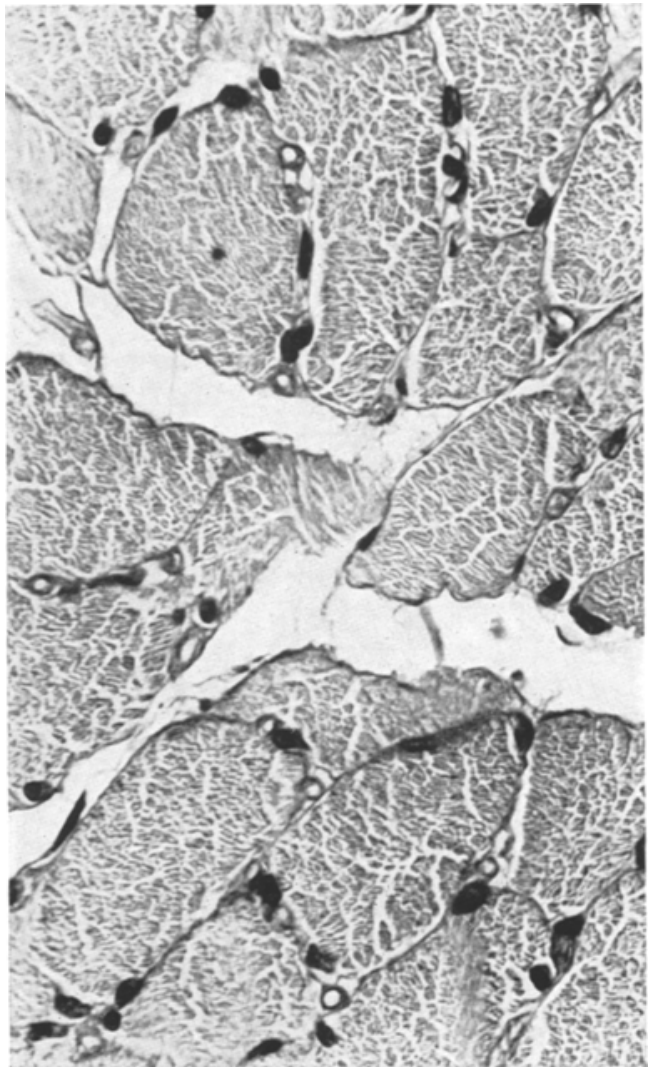

Fig. 5, Case 2. Perifocal intact muscle tissue displaying capillaropathy similar to that in Case 1 (cf. Fig. 2). (PAS staining according to McManus $\times 480$ )

\section{Discussion}

The pathological picture was essentially the same in these two cases: within a circumscribed focus, in biceps femoris in Case 1 and in vastus lateralis in Case 2, regions of haemorrhagic necrosis were found surrounded by muscle tissue that showed regressive changes and signs of regeneration. The gross and microscopical appearances agreed with those of muscular degeneration following ischaemia (cf. Adams, Denny-BRown and Pearson 1962). Therefore, when considering the pathogenesis, it is of special interest that the vessels within and outside the foci were pathologically changed. The small-vessel lesions can be ascribed to the diabetes present in both cases: the lesions agreed with those recently described as diabetic microangiopathy in muscles (Holle 1960, Zacks, Pegues and ElurOTT 1962). In Case 1 the hypertension may have con- 
tributed to the small-vessel changes (cf. BLOODworTH 1963). In both cases the coarsest arteries showed arteriosclerosis, advanced MönckmBERG's arteriosclerosis in Case 1 and atheromatosis in Case 2. Thus, both small and large vessels showed lesions that may have been of importance in the development of the muscular changes. A trauma, unnoticed or forgotten by the patient, could conceivably have served as the precipitating factor.

The microangiopathy was more pronounced in the degeneratively altered foci than in the perifocal muscle tissue. It is difficult to tell whether this difference existed before the onset of the muscular degeneration. If not, its explanation might be that anoxia enhances the development of the diabetic microangiopathy.

The observation of intraneural diabetic microangiopathy in both cases is interesting because it may signify that nerve factors were involved in the pathogenesis.

In both cases the clinical picture simulated a tumour; the reason was probably the interstitial fibrosis and oedema within the foci, as well as their distinctive demarcation. The clinical picture, with the lesions presenting as tumoriform, is quite different from that of diabetic amyotrophy (GARLAND 1955), a muscular disorder in diabetes, which has been much debated in recent years (for literature see Looke, LAWRENCE and LEGG 1963); clinically the diabetic amyotrophy appears as a wasting of muscle.

According to the clinical and pathological pictures, an appropriate term for this lesion observed in two diabetics seems to be - tumoriform focal muscular degeneration.

References. Adams, R. D., D. Denny-Brown and C.M. Pearsox: Diseases of Muscle. A Study in Pathology. Second edition. New York: Harper \& Row Publishers Inc. (1962), - BLoodworth, J.M.B., JUn.: Diabetic Microangiopathy. Diabetes, 12, 99-114(1963). - GARLAND, H.: Diabetic Amyotrophy. British Medical Journal, II 1287 (1955). - HoLLE, G.: Über elektronenmikroskopische Befunde bei diabetischer Angiopathie. Arch. Klin. Chir. 295, 253 (1960). - LOCKE, S., D. G. LAWRENCE and M. A. LEGG: Diabetic Amyotrophy. Am. J. Med. 34, 775-785 (1963). McManus, J.F.A.: Histological and Histochemical Uses of Periodic Acid. Stain Technology, 23, 99 (1948). Zacks, S.I., J.J. Pegues and F. A. Elilote: Interstitial Muscle Capillaries in Patients with Diabetes Mellitus: A Light and Electron Microscope Study. Metabolism, 11, $381-393$ (1962).

L. Argmevali

Department of Pathology II

Sahlgrenska sjukhuset

University of Gothenburg, Sweden 